Modern Physics Letters B, Vol. 10 No. 16 (1996) 785

(C) World Scientific Publishing Company

\title{
ERRATA
}

\section{OBSERVATION OF PERIODIC, QUASIPERIODIC AND CHAOTIC LOCALIZED EXCITATIONS IN NONLINEAR DIATOMIC MACRO-LATTICE}

\author{
[Mod. Phys. Lett. B, Vol. 10, Nos. 1 \& 2 (1996) 11-22] \\ SEN-YUE LOU*,†,‡, JUN YU ${ }^{\S}$, JI LIN" GUOXIANG HUANG ${ }^{\uparrow, * *}$ and JIA-KUNG ZHANG ${ }^{\S}$ \\ *Institute of Modern Physics, Ningbo Normal College, Ningbo, 315211, China \\ $\dagger$ International Centre for Theoretical Physics, P.O. Box 586, 34100 Trieste Italy \\ $¥$ Department of Physics, Fudan University, Shanghai 200433 China

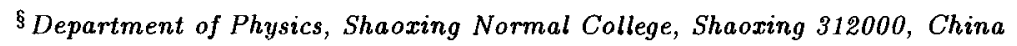 \\ "Department of Physics, Zhejiang Normal University, Jinghua 321004, China \\ I Department of Physics, East China Normal University, Shanghai 200062, China \\ ** Institute Pluridisciplinar, Universidad Complutense, \\ Paseo Juan XXIII, No. 1, Madrid 28.040, Spain
}

(1). On p. 11, the first name of the first author should be SEN-YUE.

(2). On p. 18, Eq. (8) should read

$$
A_{0}=\left[\frac{2\left(\omega_{0}-\omega_{e}+\sqrt{\delta^{2}-\gamma^{2}}\right)}{\Delta_{0}}\right]^{1 / 2}, \quad \varphi=\frac{\pi}{2}+\frac{1}{2} \sin ^{-1}\left(\frac{\gamma}{\delta}\right)
$$

${ }^{*}$ Correspondence address. 